\title{
Long-Reach Multicast High Split-Ratio Wired and Wireless WDM-PON Using SOA for Remote Upconversion
}

\author{
Lin Xu, Student Member, IEEE, Chi Wai Chow, Member, IEEE, and Hon Ki Tsang, Senior Member, IEEE
}

\begin{abstract}
We propose and experimentally demonstrate a cost-efficient hybrid gigabit wired and wireless long-reach and high split-ratio optical access network based on remote upconversion at the local exchange (LE). The scheme can deliver $40-\mathbf{G H z}$ millimeter-wave wireless services supporting wavelength multicasting over 125-km standard single-mode fiber. A semiconductor optical amplifier (SOA) is used as the multiwavelength broadband optical upconverter and optical power amplifier inside the $L E$, and a high split-ratio of 512 can be supported. Wavelength multicasting can also be realized with conversion efficiency variation of within $1 \mathrm{~dB}$ over 15-nm wavelength range. The SOA has a gain with low polarization dependency and is able to realize multiwavelength operation based on four-wave-mixing for signal upconversion. Results show that the optical millimeter-wave signal distortion due to fiber chromatic dispersion can be significantly mitigated by using the proposed scheme. Expensive RF components are not required at the $L E$ for signal upconversion because the SOA requires only dc bias. The polarization states of the input signals can be controlled at the head-end office; hence, polarization control in the LE may not be required. Error-free transmission for the wired signal was achieved and a power penalty of $2.3 \mathrm{~dB}$ was measured after $125-\mathrm{km}$ transmission.
\end{abstract}

Index Terms-Four-wave mixing (FWM), millimeter wave, radio-over-fiber (ROF), semiconductor optical amplifier (SOA).

\section{INTRODUCTION}

$\mathbf{N}$ EW BANDWIDTH (BW)-intensive services, such as high-definition television (HDTV), online gaming, and peer-to-peer networks have stimulated the deployment of passive optical networks (PONs) [1], [2]. The convergence of millimeter-wave wireless and wired PONs in an integrated platform can be a promising technique in future access networks for providing broadband wired and wireless access services with increased mobility and reduced cost [3]-[8]. The first generations of gigabit PONs (GPONs) have now been stan-

Manuscript received January 06, 2010; revised July 28, 2010; accepted July 29, 2010. Date of publication September 30, 2010; date of current version November 12, 2010. This work was supported by the Chinese University of Hong Kong (CUHK) under Group Research Grant 3110049. The work of C. W. Chow was supported under Grant NSC-98-2221-E-009-017-MY3.

L. Xu was with the Department of Electronic Engineering, Chinese University of Hong Kong, Shatin, Hong Kong. He is now with the Department of Electrical Engineering, Columbia University, New York, NY 10027 USA (e-mail: Lx2140@columbia.edu).

C. W. Chow is with the Department of Photonics, National Chiao Tung University, Hsinchu 30010, Taiwan (e-mail: cwchow@ faculty.nctu.edu.tw).

H. K. Tsang is with the Department of Electronic Engineering, Chinese University of Hong Kong, Shatin, Hong Kong (e-mail: hktsang@ ee.cuhk.edu.hk).

Color versions of one or more of the figures in this paper are available online at http://ieeexplore.ieee.org.

Digital Object Identifier 10.1109/TMTT.2010.2074830 dardized and typically offer $1-2.5-\mathrm{Gb} / \mathrm{s}$ downstream and $1-\mathrm{Gb} / \mathrm{s}$ upstream. For the wireless communication, it is now entering a new phase where the focus is moving from voice to high $\mathrm{BW}$ multimedia services. In order to support high data-rate wireless communication in the future, wireless systems operating at higher carrier frequencies, extending to the millimeter-wave regime, will be needed. Many research efforts have been made at the 60-GHz band [9]-[14] and above [15].

The generation and transmission of optical millimeter waves are crucial in radio-over-fiber (ROF) systems. Chromatic dispersion in optical fiber can cause signal distortions to the optical millimeter-wave signal. Transmission of double-sideband (DSB) optical millimeter-wave signal is degraded by a fading effect (which leads to cosine-like signal power fluctuation along the fiber) and the time shifting of data codes [16], [17]. Optical carrier suppression (OCS) optical millimeter-wave signals [16], [17] can mitigate the fading effect. However, since the upper and lower sidebands of the OCS signal carry the same data information, code time shifting (dispersion induced eye-diagram closure) is still severe after fiber propagation when the wavelength separation between the upper and lower sidebands become large. A single-sideband (SSB) optical millimeter wave can reduce the code time-shifting problem. However, the immunity depends on the generation methods, and usually the generation methods are complicated [17], [18]. An alternative approach to reduce signal distortions brought by an RF fading effect is to deliver millimeter-wave and baseband signals at different wavelengths and then mix them remotely. The remote local oscillator delivery scheme [19]-[21] is a promising deployment strategy for long-reach access networks. However, remote local oscillator delivery usually requires a costly millimeter-wave-band electrical mixer at each hybrid optical network unit (ONU) for the remote signal upconversion. Recently, several new approaches using remote signal regeneration and upconversion techniques [22] and reference signal distribution [23] have also been studied.

In this work, we propose and experimentally demonstrate a cost-efficient hybrid gigabit wired and wireless long-reach and high split-ratio access network based on remote upconversion at the local exchange (LE). The scheme can deliver 40-GHz millimeter-wave wireless services supporting wavelength multicast over a $125-\mathrm{km}$ standard single-mode fiber (SSMF). A semiconductor optical amplifier (SOA) is used as the multiwavelength broadband optical upconverter and optical power amplifier inside the LE. A high split ratio of 512 can be achieved. Wavelength multicasting can also be achieved with conversion efficiency variation of within $1 \mathrm{~dB}$ over a $15-\mathrm{nm}$ wavelength range. 


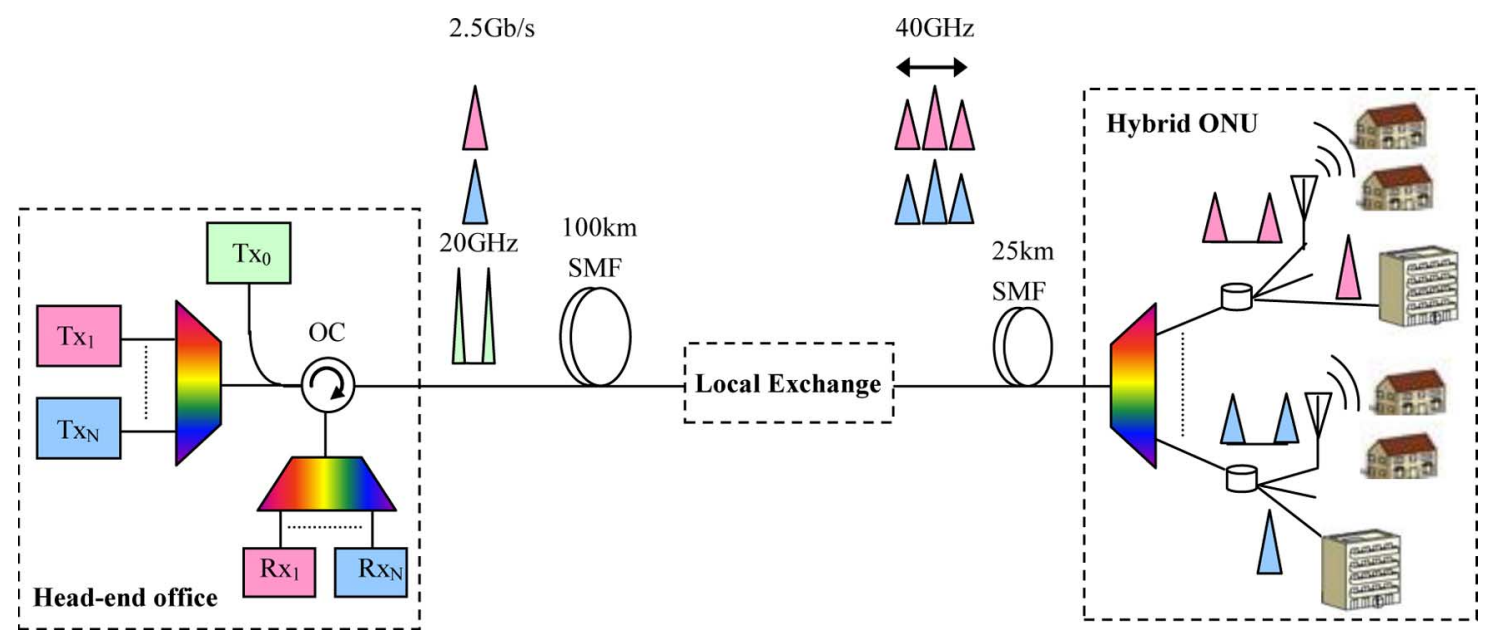

Fig. 1. Conceptual diagram of the proposed hybrid PON. Transmitter: Tx, receiver: Rx, optical circulator: OC, single mode fiber: SMF, optical network unit: ONU.

The SOA is generally considered as a potential low-cost device. It has a gain with low polarization dependency and is able to realize multiwavelength operation based on four-wave mixing (FWM) for signal upconversion. Results show that the optical millimeter-wave signal distortion due to fiber chromatic dispersion can be significantly mitigated by using the proposed scheme. Besides, expensive RF components are not required at the LE for signal upconversion because the SOA requires only dc bias. Since the polarization states of the input signals can be controlled at the head end, polarization control in the LE is not required.

Fig. 1 shows the conceptual diagram of the proposed long reach, high split-ratio wired and wireless hybrid PONs, and supporting multicasting. $2.5-\mathrm{Gb} / \mathrm{s}$ baseband signals and $20-\mathrm{GHz}$ carrier are delivered independently at different wavelengths from the head-end office. They are then mixed at the LE to generate the upconverted signal (optical millimeter-wave signal) and amplified to compensate the fiber loss. The head-end office provides the laser sources for optical signal upconversion in the wireless application and the downstream signal for the wired application. It also provides the functionality of upstream signal detection. The LE provides the optical signal frequency up-conversion and signal amplification. After the LE, two sidebands with 40-GHz spacing are generated, which both carry the $2.5-\mathrm{Gb} / \mathrm{s}$ data. In the proposed implementations, some form of polarization control will be needed (e.g., polarization controller at the LE or a polarization state monitoring in the LE with feedback to the head-end office for adjusting the polarization to maximize the conversion efficiency of the FWM). At the ONU, the center carrier is filtered for wired service detection, while the remained double sidebands are used for wireless service. Since a single LE can serve multiple users, the cost can be shared by the users. Since the upstream wireless signal can be of much lower data rate than the downstream signal, lower RF carrier frequency can be used. In this case, a direct modulation laser source, as in [24], can be used to carry the upstream ROF signal back to the head-end office. The baseband upstream data from the wired application can direct modulate a laser source or use carrier distribution [25]. In this experiment, we mainly study the downstream signal distribution and multicasting, but an upstream path has also been included.

\section{THEORETICAL BACKGROUND}

The operation of the scheme shown in Fig. 1 is explained below. For the generation of the OCS signal [see Fig. 2(d)] using a Mach-Zehnder modulator (MZM), we can consider that it consists of two phase modulators arranged in a Mach-Zehnder structure. The output $E$-field for the upper arm in the MZM is

$$
\begin{aligned}
& E_{\text {MZM-upper }}(t) \\
& \quad=\operatorname{Re}\left\{\frac{1}{\sqrt{2}} E_{0} e^{j\left(\omega_{0} t+\Delta \phi\right)}\right\} \\
& =\frac{1}{\sqrt{2}} E_{0} \cos \left(\omega_{0} t+\Delta \phi\right) \\
& =\frac{1}{\sqrt{2}} E_{0}\left\{\cos \omega_{0} t \cos \Delta \phi-\sin \omega_{0} t \sin \Delta \phi\right\}
\end{aligned}
$$

where $\omega_{0}$ and $\Delta \phi$ are the angular frequency of the input optical signal and the optical signal phase difference that is induced by the electrical applied voltage to the phase modulator, respectively. In order to simplify the analysis, we assume the applied electrical signal is sinusoidal with amplitude and frequency of $m$ and $\omega_{0}$, respectively. Hence, the voltage induced phase change to the upper phase modulator in the MZM is

$$
\Delta \phi(t)=m \cos \left(\omega_{\mathrm{RF}} t\right)
$$

Substituting (2) into (1) results in (3), shown at the bottom of the following page, where $J_{n}(m)$ is the Bessel function. By using the Bessel function, we then expand (3) and neglect the higher order $(n \geq 4)$ terms since their values are small. The output $E$-field becomes (4), shown at the bottom of the following page.

This can be illustrated in the schematic optical spectra shown in Fig. 2(a). Since the $E$-field applied to the lower phase modulator in the MZM is opposite to that of the upper phase modulator, the phase shift is negative

$$
\Delta \phi(t)=-m \cos \left(\omega_{\mathrm{RF}} t\right) .
$$


Substituting (5) into (1), results in (6), shown at the bottom of this page. Similarly, higher order $(n \geq 4)$ terms can be neglected

$$
\begin{aligned}
& E_{\mathrm{MZM}-\text { lower }}(t) \\
& \begin{aligned}
\cong \frac{1}{\sqrt{2}} E_{0}\{ & \cos \omega_{0} t\left[J_{0}(m)-2 J_{2}(m) \cos \left(2 \omega_{\mathrm{RF}} t\right)\right] \\
+ & \in \omega_{0} t\left[-2 J_{1}(m) \cos \left(\omega_{\mathrm{RF}} t\right)\right. \\
& \left.\left.+2 J_{3}(m) \cos \left(3 \omega_{\mathrm{RF}} t\right)\right]\right\} \\
=\frac{1}{\sqrt{2}} E_{0}\{ & J_{0}(m) \cos \omega_{0} t-J_{2}(m) \\
\cdot & {\left[\cos \left(\omega_{0} t+2 \omega_{\mathrm{RF}} t\right)+\cos \left(\omega_{0} t-2 \omega_{\mathrm{RF}} t\right)\right] } \\
& -J_{1}(m)\left[\sin \left(\omega_{0} t+\omega_{\mathrm{RF}} t\right)+\sin \left(\omega_{0} t-\omega_{\mathrm{RF}} t\right)\right] \\
& \left.+J_{3}(m)\left[\sin \left(\omega_{0} t+3 \omega_{\mathrm{RF}} t\right)+\sin \left(\omega_{0} t-3 \omega_{\mathrm{RF}} t\right)\right]\right\} .
\end{aligned}
\end{aligned}
$$

This can be illustrated in the schematic optical spectra shown in Fig. 2(b). When the dc bias to the $\mathrm{MZM}_{1}$ is $V_{\phi}$, the Bessel terms in (7) will rotate by $\pi$, as shown in Fig. 2(c). Fig. 2(d) then shows the combined output of the MZM.

We describe the FWM process (the signals after the LE shown in Fig. 1) with only one baseband channel (idler) for a simple explanation. The three FWM participators, as shown in Fig. 3, include pump1 at frequency $f_{1}$, pump2 at frequency $f_{2}$, and an idler at frequency $f_{3}$. Note that the component at $f_{3}$ is the down-
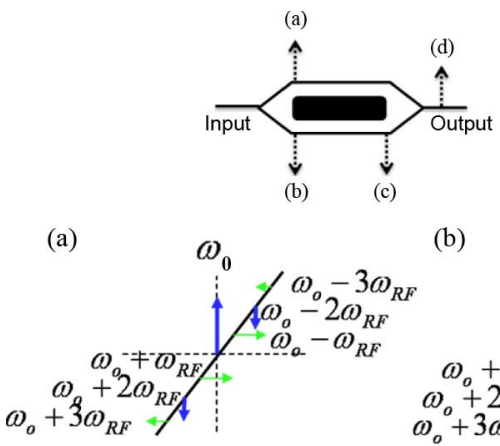

(b)
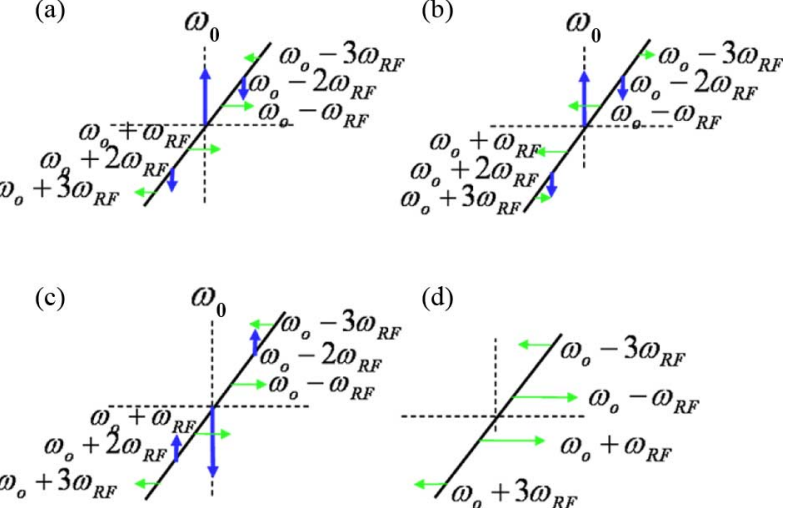

(d)

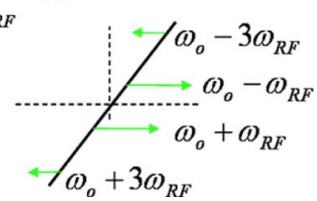

Fig. 2. (top) Schematic of the architecture of the MZM. (bottom) Schematic optical spectra at the corresponding point in the MZM.

stream baseband signal. After FWM in a nonlinear medium, new components are generated. Each generated component satisfies a frequency of $f_{x y z}=f_{x}+f_{y}-f_{z}(x, y \neq x, y, z$ are selected from 1,2, and 3) and a phase of $\phi_{x y z}=\phi_{x}+\phi_{y}-\phi_{z}$

$$
\begin{aligned}
& E_{\mathrm{MZM}-\text { upper }}(t) \\
& \quad=\frac{1}{\sqrt{2}} E_{0}\left\{\cos \omega_{0} t \cos \left[m \cos \left(\omega_{\mathrm{RF}} t\right)\right]-\sin \omega_{0} t \sin \left[m \cos \left(\omega_{\mathrm{RF}} t\right)\right]\right\} \\
& \quad=\frac{1}{\sqrt{2}} E_{0}\left\{\cos \omega_{0} t\left[J_{0}(m)+2 \sum_{n=1}^{\infty}(-1)^{n} J_{2 n}(m) \cos \left(2 n \omega_{\mathrm{RF}} t\right)\right]-\sin \omega_{0} t\left[2 \sum_{n=1}^{\infty}(-1)^{n} J_{2 n-1}(m) \cos \left[(2 n-1) \omega_{\mathrm{RF}} t\right]\right]\right\}
\end{aligned}
$$

$$
\begin{aligned}
& E_{\mathrm{MZM}-\text { upper }}(t) \\
& \begin{aligned}
\cong \frac{1}{\sqrt{2}} E_{0}\{ & \left.\cos \omega_{0} t\left[J_{0}(m)-2 J_{2}(m) \cos \left(2 \omega_{\mathrm{RF}} t\right)\right]-\sin \omega_{0} t\left[-2 J_{1}(m) \cos \left(\omega_{\mathrm{RF}} t\right)+2 J_{3}(m) \cos \left(3 \omega_{\mathrm{RF}} t\right)\right]\right\} \\
=\frac{1}{\sqrt{2}} E_{0}\{ & J_{0}(m) \cos \omega_{0} t-J_{2}(m)\left[\cos \left(\omega_{0} t+2 \omega_{\mathrm{RF}} t\right)+\cos \left(\omega_{0} t-2 \omega_{\mathrm{RF}} t\right)\right]+J_{1}(m)\left[\sin \left(\omega_{0} t+\omega_{\mathrm{RF}} t\right)+\sin \left(\omega_{0} t-\omega_{\mathrm{RF}} t\right)\right] \\
\quad & \left.\quad-J_{3}(m)\left[\sin \left(\omega_{0} t+3 \omega_{\mathrm{RF}} t\right)+\sin \left(\omega_{0} t-3 \omega_{\mathrm{RF}} t\right)\right]\right\}
\end{aligned}
\end{aligned}
$$

$$
\begin{aligned}
& E_{\mathrm{MZM}-\text { lower }}(t) \\
& \quad=\frac{1}{\sqrt{2}} E_{0}\left\{\cos \omega_{0} t \cos \left[-m \cos \left(\omega_{\mathrm{RF}} t\right)\right]-\sin \omega_{0} t \sin \left[-m \cos \left(\omega_{\mathrm{RF}} t\right)\right]\right\} \\
& =\frac{1}{\sqrt{2}} E_{0}\left\{\cos \omega_{0} t \cos \left[m \cos \left(\omega_{\mathrm{RF}} t\right)\right]+\sin \omega_{0} t \sin \left[m \cos \left(\omega_{\mathrm{RF}} t\right)\right]\right\} \\
& \quad=\frac{1}{\sqrt{2}} E_{0}\left\{\cos \omega_{0} t\left[J_{0}(m)+2 \sum_{n=1}^{\infty}(-1)^{n} J_{2 n}(m) \cos \left(2 n \omega_{\mathrm{RF}} t\right)\right]+\sin \omega_{0} t\left[2 \sum_{n=1}^{\infty}(-1)^{n} J_{2 n-1}(m) \cos \left[(2 n-1)\left(\omega_{\mathrm{RF}} t\right)\right]\right\}\right.
\end{aligned}
$$




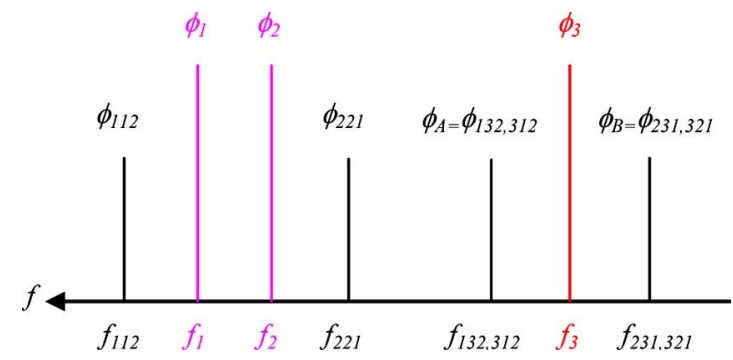

Fig. 3. Schematic diagram of FWM.

[26]. Since the two pumps $\left(f_{1}\right.$ and $\left.f_{2}\right)$ in the experiment were generated from the same laser source by OCS $\left(\phi_{1}=\phi_{2}\right)$, thus $\phi_{A}=\phi_{B}$, as shown in Fig. 3 .

By filtering the center carrier at $f_{3}$, the downstream baseband signal can be directly detected by a low-speed photodetector. Meanwhile, the optical components at $f_{132,312}$ and $f_{231,321}$ are sent to a high-speed photodetector for optical heterodyning. Assume the above two optical waves are given by

$$
\begin{aligned}
& E_{A}(t)=E_{0}(\omega t+\phi) \\
& E_{B}(t)=E_{0}^{\prime}\left(\omega^{\prime} t+\phi^{\prime}\right)
\end{aligned}
$$

where $E_{A}$ and $E_{B}$ are the amplitude terms, $\omega$ and $\omega^{\prime}$ are the angular frequency terms, and $\phi$ and $\phi^{\prime}$ are the phase terms of the two optical waves [27], [28]. The current output of a photodetector with limited BW is given by

$$
I_{\mathrm{RF}}=A \cos \left[\left(\omega-\omega^{\prime}\right)+\left(\phi-\phi^{\prime}\right)\right]
$$

where $A$ is a constant, which is determined by $E_{A}, E_{B}$, and the responsivity of the photodetector. An electrical beat note is then generated at the output of the photodetector with a frequency corresponding to the wavelength spacing of the two optical waves.

\section{EXPERIMENT}

Fig. 4 shows the proof-of-concept experiment setup for the long-reach wired and wireless hybrid PON employing a remote LE for optical upconversion. At the head-end office, two baseband signals (idlers) were generated by externally modulated tunable laser $1\left(\mathrm{TL}_{1}\right)$ at $1546.5 \mathrm{~nm}$ and tunable laser $2\left(\mathrm{TL}_{2}\right)$ at $1548 \mathrm{~nm}$. The signals were modulated using an MZM driven with $2.5 \mathrm{~Gb} / \mathrm{s}$, a pattern length of $2^{31}-1$ pseudorandom bit sequence (PRBS). A 20-GHz OCS signal (pump) was generated by externally modulated tunable laser $0\left(\mathrm{TL}_{0}\right)$ at $1545.1 \mathrm{~nm}$ using an MZM driven with a 10-GHz sinusoidal clock, acting as pump1 and pump2, shown in Fig. 3.

When the dc bias was set to a minimum transmission, OCS or DSB suppressed carrier optical modulation can be achieved. The output of the MZM has the first-order sidebands, and in principle, without the carrier frequency. The frequency separation between the two sidebands is equal to two times the modulating signal frequency. Hence, this produces frequency doubling [29]. After that, the three light waves were combined using an arrayed waveguide grating (AWG) and launched into a reel of 100-km SSMF. After transmission, three light waves were fed into an LE for remote multiwavelength upconversion. The LE consists of two optical circulators (OCs), two optical filters, two inline erbium-doped fiber amplifiers (EDFAs) for bidirectional power amplification, and an SOA. The three light waves were amplified by an EDFA. An optical filter (with 3-dB BW of $8 \mathrm{~nm}$ ) suppressed the amplified spontaneous emission (ASE) noise. The total average optical power launched into the SOA was $3.5 \mathrm{dBm}$. The SOA (Kamilian) was biased at maximum $250 \mathrm{~mA}$ in order to achieve high nonlinear effect.

The $20-\mathrm{GHz}$ clock acted as dual-pump, while baseband signals acted as idlers. Sidebands were generated with $20-\mathrm{GHz}$ separation from the center carrier of $f_{3}$ after the FWM. The signal at $1546.5 \mathrm{~nm}$ selected by another optical filter (3-dB BW of $0.5 \mathrm{~nm}$ ) was then sent to a reel of $25-\mathrm{km}$ SSMF with $10-\mathrm{dBm}$ input power. After transmission, the light was routed to the output port of an AWG depending on its wavelength. 27-dB attenuation was added using a variable optical attenuator to simulate a 512 split. A fiber Bragg grating (FBG), which has a 3-dB BW of $0.1 \mathrm{~nm}$ and extinction ratio of $20 \mathrm{~dB}$, transmitted the sidebands for wireless application and reflected the center carrier for wired application. For wireless users, the two sidebands carried with $2.5-\mathrm{Gb} / \mathrm{s}$ data will beat at photodetector and generate $40-\mathrm{GHz}$ millimeter wave. It might be pre-amplified by an electrical amplifier (EA) to boost the signal before sending it to an antenna for wireless broadband transmission. For wired users, the reflected center carrier will be directly detected.

\section{RESULTS AND DISCUSSIONS}

Fig. 5(a) shows the optical spectra of the 20-GHz DSB dual-pump and two $2.5-\mathrm{Gb} / \mathrm{s}$ idlers after $100-\mathrm{km}$ transmission. Fig. 5(b) shows the optical spectra after FWM in the SOA at the LE. Two sidebands were generated to each idler signal due to FWM. The FWM signals around the optical carrier have different amplitudes due to the combined effect of nonflat gain profile of the SOA and the filter profile used after the SOA. The separation between one sideband and the center carrier is $20 \mathrm{GHz}$, which is equal to the dual-pump separation. Since FWM is data-rate independent and capable of performing multiwavelength generation, the scheme has the potential to operate at a higher data rate and at multiple wavelengths.

The FWM conversion efficiency was also investigated, as shown in Fig. 6. The idler wavelength was detuned from the center wavelength of the dual pump while the dual pump was fixed. The inset of Fig. 6 shows the conversion efficiency. There is a small fluctuation within $1 \mathrm{~dB}$ over the $15-\mathrm{nm}$ detuning range; the tunability of the scheme can be quite wide, depending on the SOA. Note that multicasting of two wavelengths was performed in the experiment, as shown in Fig. 5, and more wavelengths may be supported simultaneously [30], [31]. Since the optical millimeter wave was transmitted all optically from the head-end office to the remote LE without carrying any data, there is only negligible phase-decorrelation induced amplitude fluctuation after $125-\mathrm{km}$ transmission [22] due to chromatic dispersion. This is mainly induced by the differential propagation delay of the two $40-\mathrm{GHz}$ spaced sidebands [32]. Fig. 7 


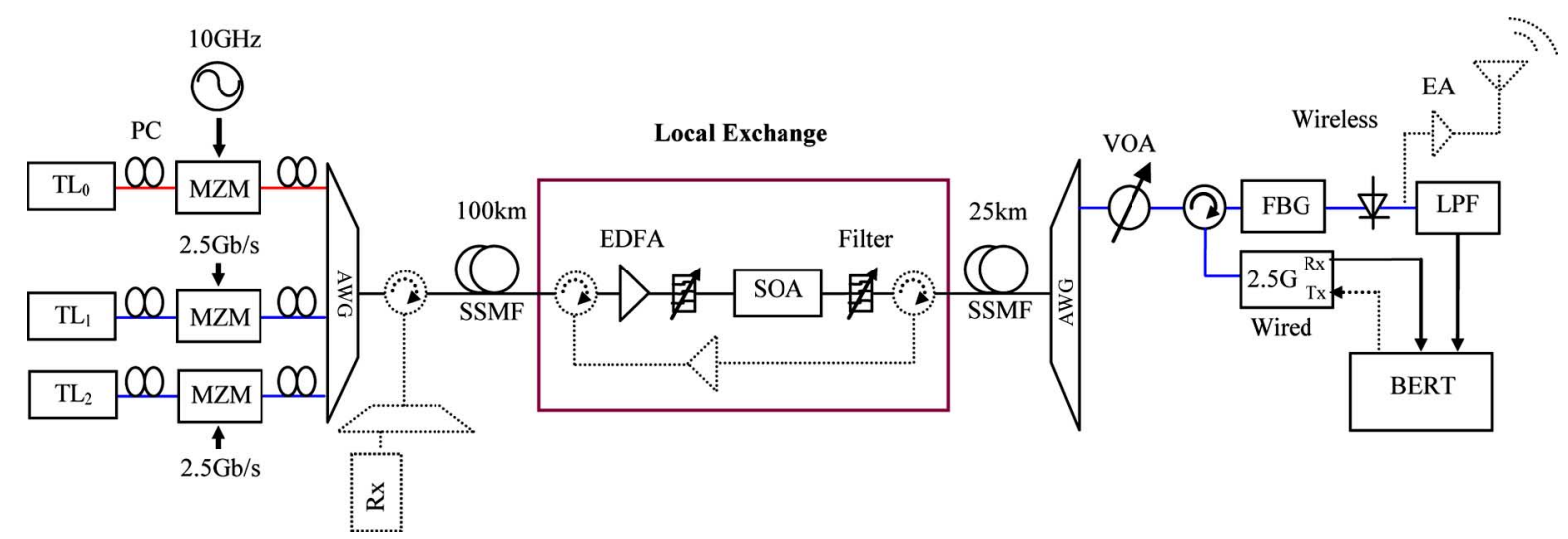

Fig. 4. Experimental setup of wired and wireless long-reach PON. Tuneable laser: TL, Mach-Zehnder modulator: MZM, arrayed waveguide grating: AWG, singlemode fiber: SMF, receiver: Rx, transmitter: Tx, polarization controller: PC, erbium-doped fiber amplifier: EDFA, fiber Bragg grating: FBG, semiconductor optical amplifier: SOA, variable optical attenuator: VOA, low-pass filter: LPF, bit-error-rate tester: BERT, electrical amplifier: EA. Dashed lines indicate the upstream path and wireless radiation, which were not demonstrated in the experiment.

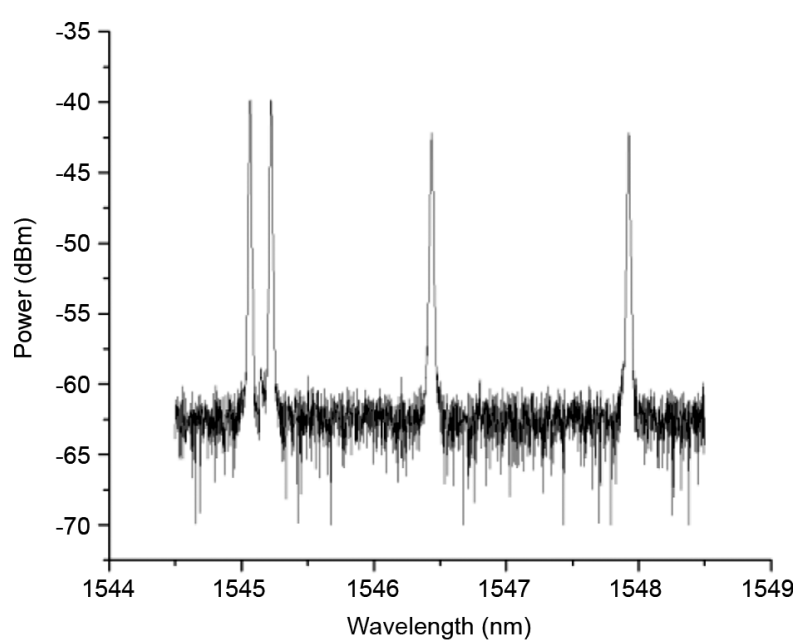

(a)

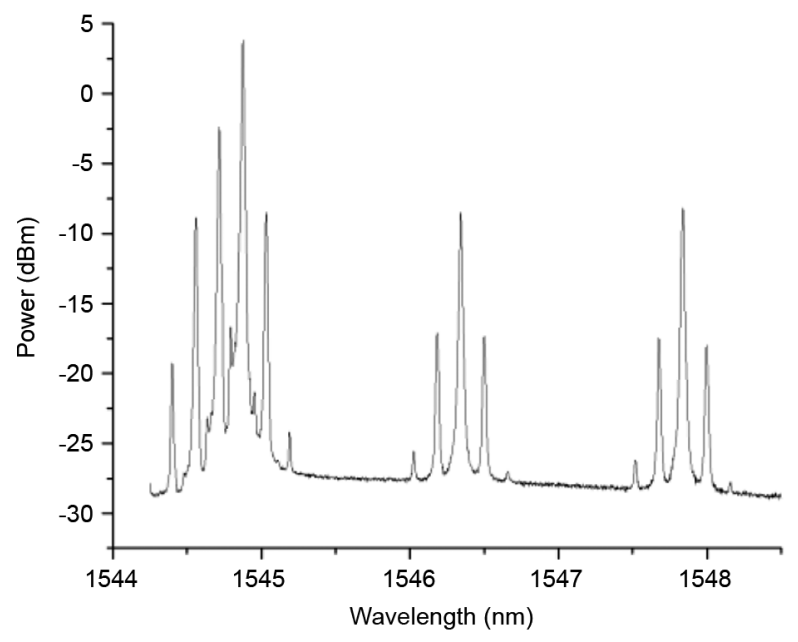

(b)

Fig. 5. Optical spectrum: (a) before and (b) after SOA.

shows the transmitted sidebands and reflected center carrier by FBG.

Fig. 8(a) shows the RF spectrum of the $40-\mathrm{GHz}$ carrier generated using two sidebands beating at a $40-\mathrm{GHz}$ photodetector followed with an RF spectrum analyzer (HP8168). Its resolution $\mathrm{BW}$ was $100 \mathrm{kHz}$. A carrier-to-noise ratio of $30 \mathrm{~dB}$ was

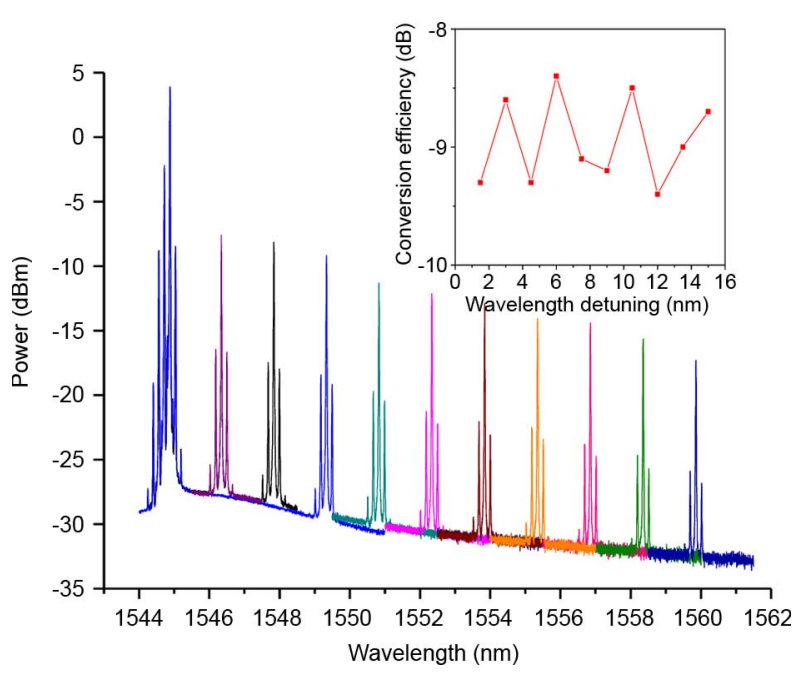

Fig. 6. FWM conversion efficiency.

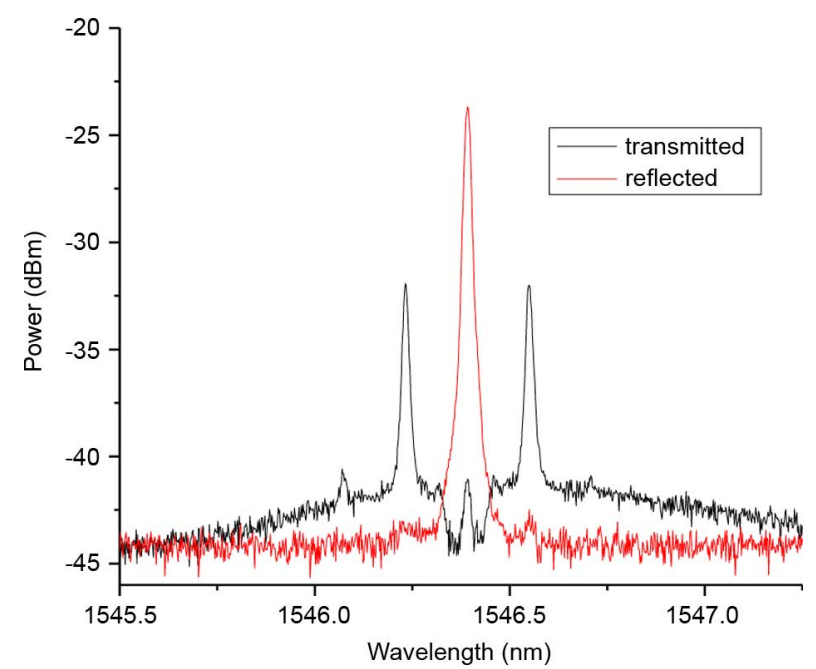

Fig. 7. Optical spectrum of transmitted and reflected light by FBG.

observed in the measurement. The 40-GHz clock signal was obtained when baseband data modulation was turned off in order to compare the performance of the generated clock signal with that 


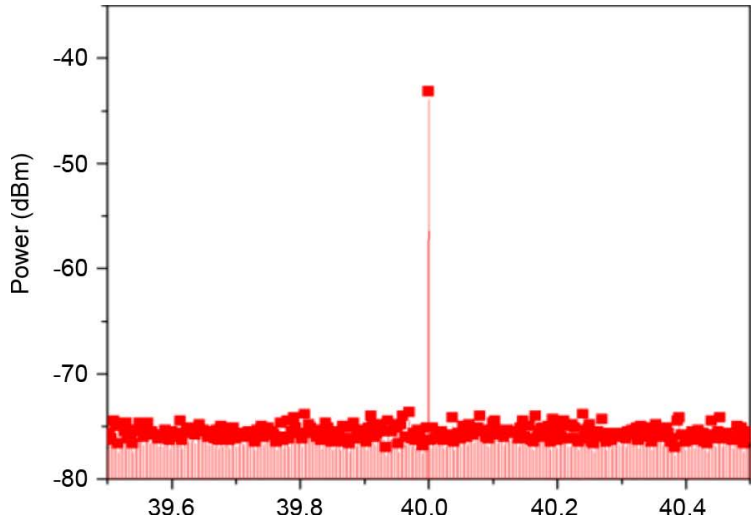

(a)

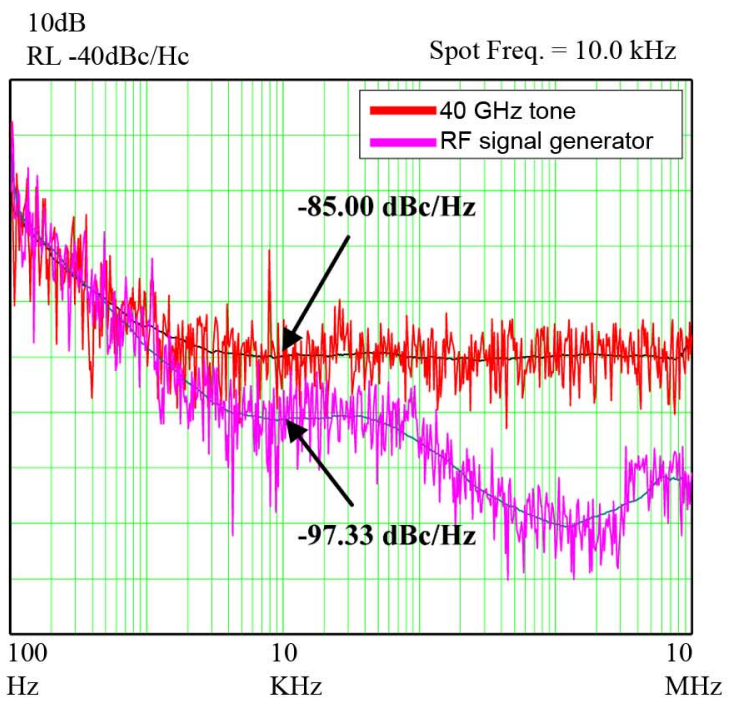

(b)

Fig. 8. $40 \mathrm{GHz}$ : (a) RF spectrum and (b) phase noise.

directly from the synthesizer. The phase noise was also measured to analyze the performance of the measured $40-\mathrm{GHz}$ carrier, as shown in Fig. 8(b). The purple line (in online version) indicates the phase noise of the $40-\mathrm{GHz}$ signal generated at the source RF signal generator used in the experiment setup. The red line (in online version) shows the phase noise of the $40-\mathrm{GHz}$ carrier obtained using the proposed method. As shown in Fig. 8(b), the phase noise of the 40-GHz carrier from the proposed scheme was $\sim 12 \mathrm{~dB}$ higher than that of the RF signal generator at the frequency offset of $10 \mathrm{kHz}$.

The measured RF spectrum of the $2.5-\mathrm{Gb} / \mathrm{s}$ wireless signal is shown in Fig. 9. The inset is the eye diagrams after passing the 7.73-GHz low-pass filter. We can observe that a clear eye opening can be achieved in the long-reach network by using the proposed scheme. Note here that the back-to-back (B2B) case is at $100-\mathrm{km}$ transmission.

Since we did not have a 40-GHz RF mixer and RF filter, numerical analysis (using VPI Transmission MakerV7.5) was performed to evaluate the wireless signal. In the simulation, a DSB (with carrier) signal was generated, carrying $2.5-\mathrm{Gb} / \mathrm{s}$ nonreturn-to-zero (NRZ) data on both sidebands. The separation between one sideband to the center carrier was $20 \mathrm{GHz}$. The signal

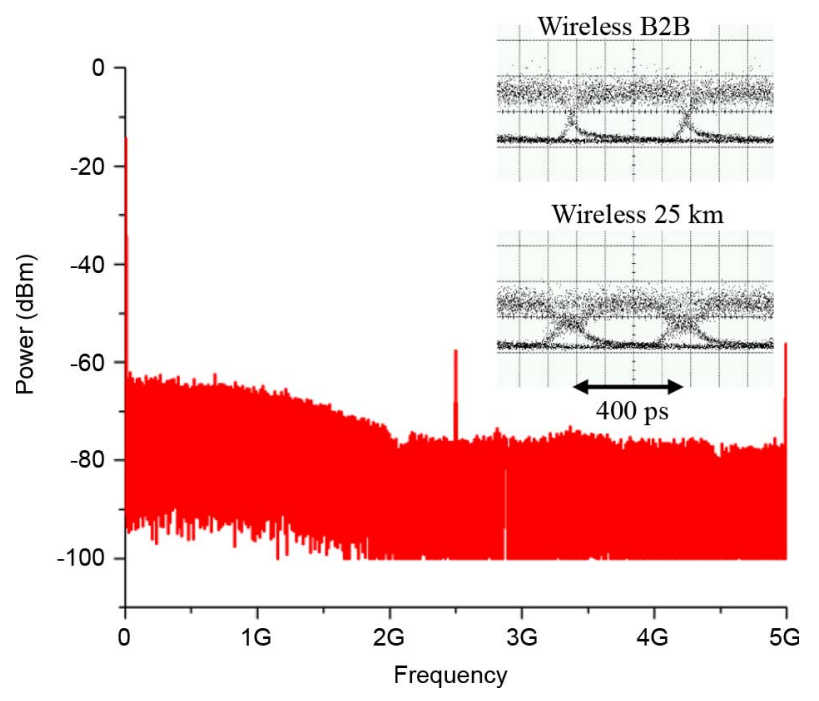

Fig. 9. Measured RF spectrum of wireless $2.5-\mathrm{Gb} / \mathrm{s}$ signal. Inset: eye diagrams of the wireless $2.5-\mathrm{Gb} / \mathrm{s}$ signal.

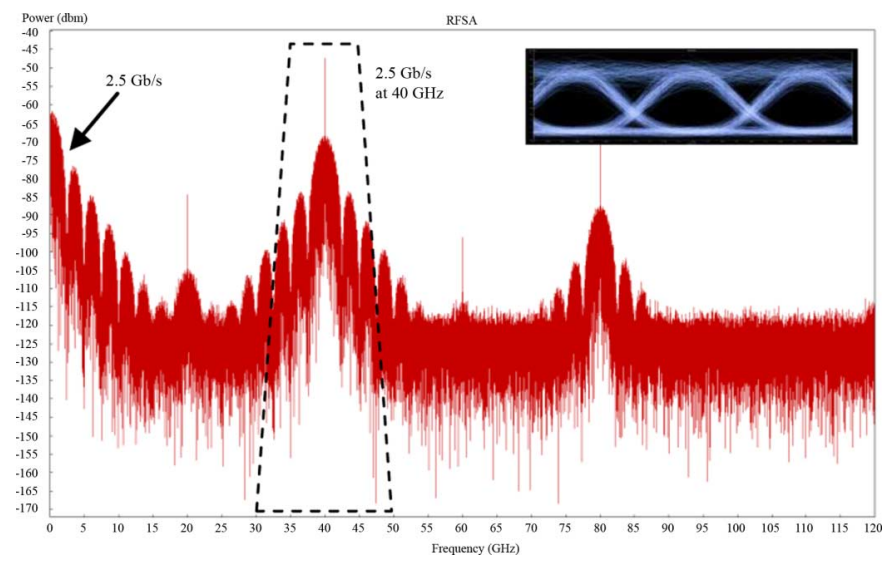

Fig. 10. Simulated RF spectrum of $2.5-\mathrm{Gb} / \mathrm{s}$ signal on $40-\mathrm{GHz}$ carrier and respective eye diagram.

was transmitted in SSMF (dispersion parameter: $17 \mathrm{ps} / \mathrm{nm} / \mathrm{km}$ ). A FBG with the transmission profile similar to the experimental one was used in the simulation. After the FBG, DSBs with a carrier suppression optical millimeter-wave signal was generated. Fig. 10 shows the simulated RF spectrum of the optical millimeter wave detected after the FBG (Fig. 2). We can observe that the dominant signal is the $2.5-\mathrm{Gb} / \mathrm{s}$ data at the frequency band of $40 \mathrm{GHz}$. An electrical bandpass filter, or an RF antenna with a suitable passband, can be used to extract this signal for wireless applications. In the simulation, the wireless signal was extracted by a Bessel bandpass filter (center frequency at $40 \mathrm{GHz}$ and $3-\mathrm{dB} \mathrm{BW}$ of $5 \mathrm{GHz}$ ) and then RF down-converted to the baseband by using a 40-GHz electrical sinusoidal signal. The output port of the RF mixer was followed by a Bessel low-pass filter with 3-dB BW of $2.5 \mathrm{GHz}$. The inset of Fig. 10 shows the simulated $40-\mathrm{GHz}$ down-converted $2.5-\mathrm{Gb} / \mathrm{s}$ eye diagram with a clear eye opening.

We can also observe from Fig. 10 that baseband $2.5-\mathrm{Gb} / \mathrm{s}$ data also appeared due to the self-beating of each sideband of the OCS signal. A Bessel low-pass filter was used to detect this signal. Fig. 11(a) and (b) shows the simulated eye diagrams of 
(a)

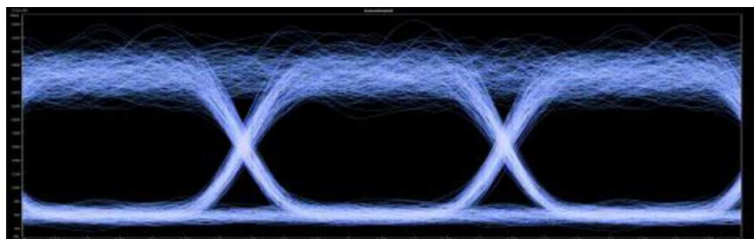

(b)

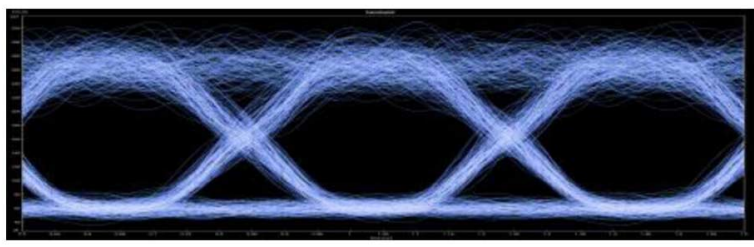

(c)

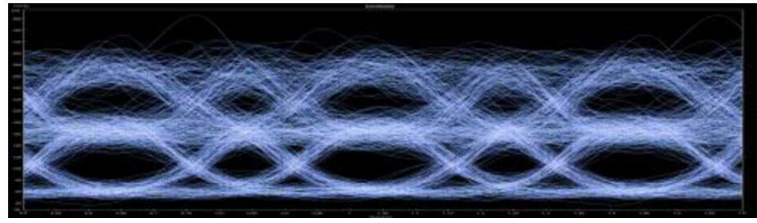

Fig. 11. Simulated eye diagrams for $2.5-\mathrm{Gb} / \mathrm{s}$ signal on $40-\mathrm{GHz}$ carrier (a) Back-to back. (b) After 25-km transmission. (c) After 125-km transmission.

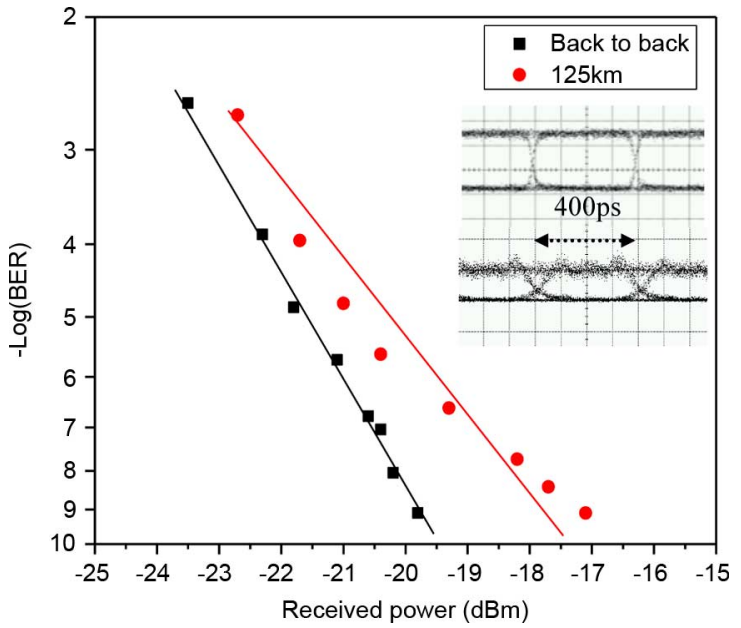

Fig. 12. Experimental BER and eye diagrams for $2.5-\mathrm{Gb} / \mathrm{s}$ wired signal.

the 2.5-Gb/s signals at B2B and after the 25-km SSMF transmission. We can see that there is a good match in eye shape with the experimental signals shown in the insets of Fig. 8. We can observe that the $25-\mathrm{km}$ fiber chromatic dispersion causes the reduction in eye width, which can also be observed in Fig. 11(b). Finally, we also numerically studied the case in which the proposed scheme of the optical upconversion at the LE was not deployed, and an optical millimeter-wave signal (2.5-Gb/s NRZ data carried by an optical OCS signal with sideband separation of $40 \mathrm{GHz}$ ) was propagating in 125-km SSMF. Fig. 11(c) shows the optical millimeter-wave signal after 125-km SSMF, showing that the signal can hardly be detected. Results show that the proposed scheme can significantly mitigate the fiber chromatic dispersion distortion introduced to the ROF signal.

Fig. 12 shows the BER performance and corresponding eye diagrams for the wired applications. Note that the B2B case here is at the head-end office. The receiver sensitivity and power penalty without pre-amplification are $-17.5 \mathrm{dBm}$ and $2.3 \mathrm{~dB}$ at $10^{-9} \mathrm{BER}$, respectively.

\section{CONCLUSIONS}

We have proposed and experimentally demonstrated a hybrid gigabit wired and wireless long-reach and high split-ratio access network based on remote upconversion at the LE. The scheme can deliver 40-GHz millimeter-wave wireless services supporting wavelength multicast over $125-\mathrm{km}$ SSMF. Only one SOA is needed as the multiwavelength broadband optical upconverter and optical power amplifier inside the LE, and a high split ratio of 512 can be achieved. Wavelength multicast can also be achieved with conversion efficiency variation of within $1 \mathrm{~dB}$ over the 15-nm wavelength range. The SOA at the LE may support multiwavelength operation based on FWM for signal upconversion. The experimental results show that the optical millimeter-wave signal distortion due to fiber chromatic dispersion can be significantly mitigated by using the proposed scheme.

\section{REFERENCES}

[1] T. Koonen, "Fiber to the home/fiber to the premises: What, where, and when?," Proc. IEEE, vol. 94, no. 5, pp. 911-934, May 2006.

[2] C. H. Lee et al., "Fiber to the home using a PON infrastructure," $J$. Lightw. Technol., vol. 24, no. 12, pp. 4568-4583, Dec. 2006.

[3] T. Kamisaka, T. Kuri, and K. Kitayama, "Simultaneous modulation and fiber-optic transmission of $10 \mathrm{~Gb} / \mathrm{s}$ baseband and $60 \mathrm{GHz}$ band radio signals on a single wavelength," IEEE Trans. Microw. Theory Tech., vol. 49, no. 10, pp. 2013-2017, Oct. 2001.

[4] K. Ikeda, T. Kuri, and K. Kitayama, "Simultaneous three band modulation and fiber-optic transmission of $2.5 \mathrm{~Gb} / \mathrm{s}$ baseband, microwave, and $60 \mathrm{GHz}$ band signals on a single wavelength," J. Lightw. Technol., vol. 21, no. 12, pp. 3194-3202, Dec. 2003.

[5] A. Martinez, V. Polo, and J. Marti, "Simultaneous baseband and RF optical modulation scheme for feeding wireless and wireline heterogeneous access network," IEEE Trans. Microw. Theory Tech., vol. 49, no. 10, pp. 2018-2024, Oct. 2001.

[6] G. K. Chang, J. Yu, Z. Jia, and J. Yu, "Novel optical-wireless access network architecture for simultaneously providing broadband wireless and wired services," in Proc. Opt. Fiber Commun., Anaheim, CA, 2006, Paper OFMI.

[7] C. T. Lin, W. R. Peng, P. C. Peng, J. Chen, C. F. Peng, B. S. Chiou, and $\mathrm{S}$. Chi, "Simultaneous generation of baseband and radio signals using only one single electrode Mach-Zehnder modulator with enhanced linearity," IEEE Photon. Technol. Lett., vol. 18, no. 23, pp. 2481-2483, Dec. 2006.

[8] C. T. Lin, J. Chen, P. C. Peng, C. F. Peng, W. R. Peng, B. S. Chiou, and S. Chi, "Hybrid optical access network integrating fiber-to-the-home and radio-over-fiber systems," IEEE Photon. Technol. Lett., vol. 19, no. 8, pp. 610-612, Apr. 2007.

[9] J. J. V. Olmos, T. Kuri, and K.-I. Kitayama, "Dynamic reconfigurable WDM $60 \mathrm{GHz}$ millimeter-wave-band radio-over-fiber access network: Architectural considerations and experiment," J. Lightw. Technol, vol. 25, no. 11, pp. 3374-3380, Nov. 2007.

[10] M. Huchard, M. Weiss, A. Pizzinat, S. Meyer, P. Guignard, and B. Charbonnier, "Ultra-broadband wireless home network based on 60-GHz WPAN cells interconnected via RoF," J. Lightw. Technol., vol. 26, no. 15, pp. 2364-2372, Aug. 2008.

[11] J. Kim, Y.-D. Chung, K.-S. Choi, D.-S. Shin, J.-S. Sim, and H.-K. $\mathrm{Yu}$, "60-GHz system-on-packaging transmitter for radio-over-fiber applications," J. Lightw. Technol., vol. 26, no. 15, pp. 2379-2387, Aug. 2008.

[12] M. Weiß, M. Huchard, A. Stöhr, B. Charbonnier, S. Fedderwitz, and D. Jäger, "60-GHz photonic millimeter-wave link for short-to mediumrange wireless transmission up to $12.5 \mathrm{~Gb} / \mathrm{s}$," J. Lightw. Technol., vol. 26, no. 15, pp. 2424-2429, Aug. 2008.

[13] J. J. V. Olmos, T. Kuri, and K.-I. Kitayama, "Dynamic reconfigurable WDM 60 Ghz millimeter-wave-band radio-over-fiber access network: architectural considerations and experiment," J. Lightw. Technol., vol. 25, no. 11, pp. 3374-3380, Nov. 2007.

[14] J. J. V. Olmos, T. Kuri, T. Sono, K. Tamura, H. Toda, and K.-I. Kitayama, "Reconfigurable 2.5-Gb/s baseband and $60-\mathrm{GHz}(155-\mathrm{Mb} / \mathrm{s})$ millimeter-waveband radio-over-fiber (interleaving) access network," J. Lightw. Technol., vol. 26, no. 15, pp. 2506-2512, Aug. 2008. 
[15] A. Hirata, H. Takahashi, R. Yamaguchi, T. Kosugi, K. Murata, T. Nagatsuma, N. Kukutsu, and Y. Kado, "Transmission characteristics of 120-GHz-band wireless link using radio-on-fiber technologies," $J$. Lightw. Technol., vol. 26, no. 15, pp. 2338-2344, Aug. 2008.

[16] J. Yu, M. F. Huang, Z. Jia, T. Wang, and G. K. Chang, "A novel scheme to generate single-sideband millimeter-wave signals by using low-frequency local oscillator signal," IEEE Photon. Technol. Lett., vol. 20, no. 7, pp. 478-480, Apr. 2008.

[17] J. Ma, J. Yu, C. Yu, X. Xin, J. Zeng, and L. Chen, "Fiber dispersion influence on transmission of the optical millimeter-wave generated using LN-MZM intensity modulation," J. Lightw. Technol., vol. 25, no. 11, pp. 3244-3256, Nov. 2007.

[18] C.-T. Lin, Y.-M. Lin, J. Chen, S.-P. Dai, P. T. Shih, P.-C. Peng, and S. Chi, "Optical direct-detection OFDM signal generation for radio-overfiber link using frequency doubling scheme with carrier suppression," Opt. Exp., vol. 16, no. 9, pp. 6056-6063, 2008.

[19] C. Lim, A. Nirmalathas, D. Novak, R. Waterhouse, and G. Yoffe, "Millimeter-wave broadband fiber-wireless system incorporating baseband data transmission over fiber and remote LO delivery," $J$. Lightw. Technol., vol. 18, no. 10, pp. 1355-1363, Oct. 2000.

[20] T. Ismail, C. P. Liu, and A. J. Seeds, "Millimetre-wave Gigabit/s wireless-over-Fibre transmission using low cost uncooled devices with remote local oscillator delivery," in Proc. OFC/NFOEC, 2007, Paper OWN3.

[21] S. A. Malyshev and A. L. Chizh, "p-i-n photodiodes for frequency mixing in radio-over-fiber systems," J. Lightw. Technol., vol. 25, no. 11, pp. 3236-3243, Nov. 2007.

[22] H. C. Chien, A. Chowdhury, Z. Jia, Y. T. Hsueh, and G. K. Chang, "60 GHz millimeter-wave gigabit wireless services over long-reach passive optical network using remote signal regeneration and upconversion," Opt. Exp., vol. 17, no. 5, pp. 3036-3041, Mar. 2009.

[23] C. W. Chow, L. Xu, C. H. Yeh, C. H. Wang, F. Y. Shih, H. K. Tsang, C. L. Pan, and S. Chi, "Mitigation of signal distortions using reference signal distribution with colorless remote antenna units for radio-overfiber applications," J. Lightw. Technol., vol. 27, no. 21, pp. 4773-4780, Nov. 2009

[24] J. B. Jensen, T. B. Gibbon, X. Yu, R. Rodes, and I. T. Monroy, "Bidirectional $3.125 \mathrm{Gbps}$ downstream/2 Gbps upstream impulse radio ultrawide-band (UWB) over combined fiber and wireless link," in Proc. OFC, 2010, Paper OThO5.

[25] J. He, L. Chen, Z. Dong, S. Wen, and J. Yu, "Full-duplex radio-over-fiber system with photonics frequency quadruples for optical millimeter-wave generation," Opt. Fiber Technol., vol. 15, pp. 290-295, 2009.

[26] N. Shibata, R. P. Braun, and R. G. Waarts, "Phase-mismatch dependence of efficiency of wave generation through four-wave mixing in a single-mode optical fiber," IEEE J. Quant. Electron., vol. QE-23, no. 7, pp. 1205-1210, Jul. 1987.

[27] U. Gliese, T. N. Nielsen, S. Nørskov, and K. E. Stubkjaer, "Multifunctional fiber-optic microwave links based on remote heterodyne detection," IEEE Trans. Microw. Theory Tech., vol. 46, no. 5, pp. 458-468, May 1998

[28] J. Yao, "Microwave photonics," J. Lightw. Technol., vol. 27, no. 3, pp. 314-335, Feb. 2009

[29] L. Xu, C. Li, C. W. Chow, and H. K. Tsang, "Optical mm-wave signal generation by frequency quadrupling using an optical modulator and a silicon microresonator filter," IEEE Photon. Technol. Lett., vol. 21, no. 2, pp. 209-211, Feb. 2009.

[30] H. Wen, H. Jiang, X. Zheng, H. Zhang, and Y. Guo, "Performance enhancement of multiwavelength conversion of RZ-DPSK based on four-wave mixing in semiconductor optical amplifier," IEEE Photon. Technol. Lett., vol. 19, no. 9, pp. 1377-1379, Sep. 2007.
[31] S. Fu, W. D. Zhong, P. Shum, Y. J. Wen, and M. Tang, "Simultaneous multichannel photonic up-conversion based on nonlinear polarization rotation of an SOA for radio-over-fiber systems," IEEE Photon. Technol. Lett., vol. 21, no. 5, pp. 563-565, May 2009.

[32] U. Gliese, S. Norskov, and T. N. Nielsen, "Chromatic dispersion in fiber-optic microwave and millimeter-wave links," IEEE Trans. Microw. Theory Tech., vol. 44, no. 10, pp. 1716-1724, Oct. 1996.

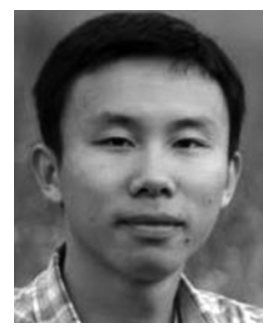

Lin Xu (S'08) received the B.Sc. degree from the Huazhong University of Science and Technology, Wuhan, China, in 2006, and the Ph.D. degree in electronic engineering from the Chinese University of Hong Kong, Shatin, Hong Kong, in 2010.

In 2009, he was a Visiting Scholar with the Photonics Research Group, Cambridge University, Cambridge, U.K. He is currently a Postdoctoral Research Scientist with Columbia University, New York, NY. His research interests are novel phonics devices and subsystems for access networks.

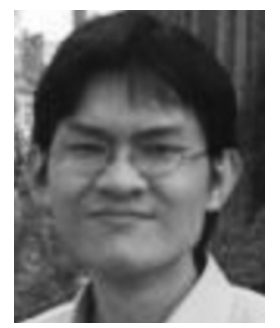

Chi Wai Chow (M'04) received the B.Eng. (firstClass Hons) and Ph.D. degrees in electronic engineering from the Chinese University of Hong Kong, Shatin, Hong Kong, in 2001 and 2004, respectively. His doctoral dissertation focused on photonic packet switching.

He was a Postdoctoral Fellow with the Chinese University of Hong Kong (CUHK), Shatin, Hong Kong, where he was involved with hybrid integration and silicon photonics. From 2005 to 2007, he was a Postdoctoral Research Scientist with the Tyndall National Institute and Department of Physics, University College Cork (UCC), Cork, Ireland, where he was primarily invovled with two European Union Projects: PIEMAN and TRIUMPH. In 2007, he joined the Department of Photonics, National Chiao Tung University, Hsinchu, Taiwan, as an Assistant Professor. His research interests are PONs, ROF, and photonic signal processing.

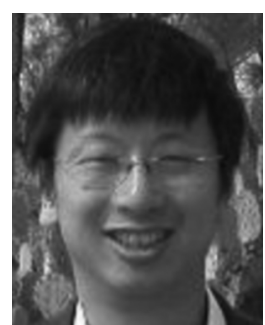

Hon Ki Tsang (M'91-SM'04) received the B.A. degree (Hons) in engineering (electrical and information sciences) and Ph.D. and M.A. degrees from the University of Cambridge, Cambridge, U.K., in 1987 and 1991 .

From 1991 to 1993, he was an SERC Postdoctoral Fellow with the University of Bath. In 1993, he joined the Chinese University of Hong Kong, Shatin, Hong Kong, as a Lecturer with the Department of Electronic Engineering. From 2002 to 2003, he was Director of ASOC Technology at Bookham Technology, Oxfordshire, U.K. In 2003, he returned to the Chinese University of Hong Kong, where he is currently Chairman of the Department of Electronic Engineering. He has authored or coauthored over 230 papers in journals or conference proceedings.

Dr. Tsang was a chair of the IEEE Lasers and Electro-Optics Society (LEOS) Hong Kong Chapter. He is currently an associate editor of the IEEE Photonics Society Newsletter. He serves on the Technical Program Committees of various conferences. 